Bull. Korean Math. Soc. 50 (2013), No. 2, pp. 543-558

http://dx.doi.org/10.4134/BKMS.2013.50.2.543

\title{
ON RIGHT-ANGLED ARTIN GROUPS WHOSE UNDERLYING GRAPHS HAVE TWO VERTICES WITH THE SAME LINK
}

\author{
Jongtae Kim and Myoungho Moon
}

\begin{abstract}
Let $\Gamma$ be a graph which contains two vertices $a, b$ with the same link. For the case where the link has less than 3 vertices, we prove that if the right-angled Artin group $A(\Gamma)$ contains a hyperbolic surface subgroup, then $A(\Gamma-\{a\})$ contains a hyperbolic surface subgroup. Moreover, we also show that the same result holds with certain restrictions for the case where the link has more than or equal to 3 vertices.
\end{abstract}

\section{Introduction}

Let $\Gamma$ be a finite simple graph with the vertex set $V(\Gamma)=\left\{v_{1}, \ldots, v_{n}\right\}$. Recall that a finite simple graph is a finite graph without loops and multiple edges. In a finite simple graph, for a pair of two vertices, there is at most one edge having them as endpoints. A right-angled Artin group $A(\Gamma)$ on the graph $\Gamma$ is the group given by the presentation with generators $v_{1}, \ldots, v_{n}$ and defining relators $\left[v_{i}, v_{j}\right]$ whenever there is an edge having $v_{i}$ and $v_{j}$ as endpoints. The graph $\Gamma$ is called the defining graph of $A(\Gamma)$. It is known that two right-angled Artin groups are isomorphic if and only if their defining graphs are isomorphic as graphs $([6])$.

A subgraph $\Gamma^{\prime}$ of a simple graph $\Gamma$ is called an induced subgraph if for each pair of vertices $a, b$ of $\Gamma^{\prime}$, there is an edge having $a$ and $b$ as endpoints in $\Gamma^{\prime}$ whenever there is an edge having $a$ and $b$ as endpoints in $\Gamma$.

Note that for a subset $S$ of the vertex set of a graph $\Gamma$, there is a unique induced subgraph of $\Gamma$ having $S$ as the vertex set. This induced subgraph is called the subgraph generated by $S$ and denoted by $\langle S\rangle$. For a subset $\left\{v_{1}, \ldots, v_{m}\right\}$ of vertices in $\Gamma$, we will denote the induced subgraph generated by $V(\Gamma)-$ $\left\{v_{1}, \ldots, v_{m}\right\}$ by $\Gamma-\left\{v_{1}, \ldots, v_{m}\right\}$. It can be shown that if $\Gamma^{\prime}$ is an induced subgraph of a graph $\Gamma$, then $A\left(\Gamma^{\prime}\right)$ is isomorphic to a subgroup of $A(\Gamma)$. More precisely, if $\Gamma^{\prime}$ is an induced subgraph of a graph $\Gamma$, then $A\left(\Gamma^{\prime}\right)$ is isomorphic to the subgroup generated by $V\left(\Gamma^{\prime}\right)$ in $A(\Gamma)$ (for a proof, see [8]).

Received November 25, 2011.

2010 Mathematics Subject Classification. Primary 20F36, 20F65; Secondary 05C25.

Key words and phrases. right-angled Artin group, hyperbolic surface subgroup. 
Motivated by the virtual Haken conjecture in 3-manifold theory, it has been an intriguing question to ask whether given a graph $\Gamma$ the right-angled Artin group $A(\Gamma)$ contains the fundamental group of a closed orientable hyperbolic surface or not $([4,5,9,10,12])$. For convenience, we will call the fundamental group of a closed orientable hyperbolic surface by a hyperbolic surface group.

An $n$-cycle, denoted by $C_{n}$, is the graph that is homeomorphic to the circle with $n$ edges. H. Servatius, C. Droms, and B. Servatius showed that if $\Gamma$ contains an induced $n$-cycle with $n \geq 5$, called a long cycle, then $A(\Gamma)$ contains a hyperbolic surface group $([12])$. S. Kim [9] showed that there is a graph $\Gamma$ such that $A(\Gamma)$ contains a hyperbolic surface group although $\Gamma$ does not contain any long cycle. A graph is called chordal if there is no induced $n$-cycle with $n \geq 4$ in the entire graph. He also showed that if a graph $\Gamma$ is chordal, then the right-angled Artin group on $\Gamma$ does not contain a hyperbolic surface subgroup $([10])$.

In [4], Crisp, Sageev, and Sapir found eight special graphs with less than or equal to 8 vertices and gave a description of a computer based proof that every graph $\Gamma$ with less than or equal to 8 vertices contains either one of the eight special graphs or a long cycle as an induced subgraph if and only if $A(\Gamma)$ contains a hyperbolic surface group.

In that paper, they raised several questions on right-angled Artin groups which contain a hyperbolic surface group. Among them, we will discuss on Questions 1.1 and 1.2 below. They are reasonable questions to ask, because the problem of determining whether the right-angled Artin group on a given graph contains a hyperbolic surface group can be reduced to a simpler graph case if the answers to them are yes.

Question 1.1 ([4]). Suppose a graph $\Gamma$ contains two vertices $a, b$ with the same links and $A(\Gamma)$ contains a hyperbolic surface subgroup. Does $A(\Gamma-\{a\})$ contain a hyperbolic surface subgroup?

Recall that for a vertex $v$ in a graph $\Gamma$, the set of vertices adjacent to $v$ is called the $\operatorname{link}$ of $v$ and denoted by $\operatorname{link}(v)$. Note that $v$ itself is not in the link of $v$. The main purpose of this paper is to provide a positive answer to this question when the link has less than or equal to 4 vertices with certain restrictions.

Question $1.2([4])$. Suppose that a graph $\Gamma$ is an amalgamation of two proper subgraphs $\Gamma_{1}, \Gamma_{2}$ along a complete graph $L$ and the right-angled Artin group $A(\Gamma)$ contains a hyperbolic surface subgroup. Is it true that $A\left(\Gamma_{1}\right)$ or $A\left(\Gamma_{2}\right)$ also contain a hyperbolic surface subgroup?

A complete graph is a simple graph each of whose vertices is adjacent to all the other vertices. It is not known whether the answer for this question is yes or no. In [4], Crisp, Sageev, and Sapir mentioned that the question can be answered affirmatively for one vertex amalgamation case, and they omitted a proof for that result. We give a detailed proof for one vertex amalgamation 
case, and use it to obtain the results regarding Question 1.1. Through a careful observation, we obtain a similar result when $L$ is not complete but has the property that every vertex has the same link (see Theorem 3.4).

A dissection on a compact surface, established by Crisp and Wiest [5], is a geometric tool to deal with homomorphisms from hyperbolic surface groups to right-angled Artin groups. In Section 2, a part of the basic cutting lemma on dissections by Crisp, Sageev, and Sapir in [4] will be proved. In Section 3, we will discuss on Question 1.1 and Question 1.2.

In order to answer Question 1.1 and Question 1.2, we only need to consider the case where $\Gamma$ is connected. In fact, if $\Gamma$ is not connected, then $A(\Gamma)$ is the free product of the right-angled Artin groups on the components of $\Gamma$. Since hyperbolic surface groups are indecomposable, the right-angled Artin group on one of the components of $\Gamma$ contains a hyperbolic surface group, if $A(\Gamma)$ does. Thus we assume that the graph $\Gamma$ is connected throughout the paper. This paper is an improved version of a part of the doctoral dissertation of the first author.

\section{Basic cutting lemma}

Let $S$ be a compact orientable surface and $\partial S$ be its boundary (maybe empty). Recall that a properly embedded arc is the image of a closed interval into $S$ by an embedding such that the image of the two endpoints of the interval are in $\partial S$. Let $\alpha$ be a simple closed curve or a properly embedded arc. A transverse orientation of $\alpha$ on $S$ is a choice of one of two components of the total space of the normal bundle of $\alpha$ in $S$. A transverse orientation of $\alpha$ on $S$ can be simply indicated by a short arrow crossing $\alpha$ from one side to the other in a tubular neighborhood of $\alpha$. Transversely oriented simple closed curves, transversely oriented properly embedded arcs, and transversely oriented boundary components of $S$ are called collectively hypercurves in $S$ ([5]). Note that if $S$ is closed, then all hypercurves are transversely oriented simple closed curves.

For two properly embedded arcs $\alpha$ and $\beta$, we say $\alpha$ and $\beta$ are homotopic if $\alpha$ and $\beta$ are homotopic in the usual sense by a homotopy leaving the endpoints of $\alpha$ and $\beta$ on the boundary of $S$ (but not requiring the endpoints to be fixed). We say an arc (or a curve) $\alpha$ is homotopic into $\partial S$ if there is an arc (or a curve) $\beta$ in $\partial S$ (freely if $\alpha$ is a curve) homotopic to $\alpha$. Two hypercurves on a surface $S$ are said to intersect transversely, if they meet in a discrete set of points and for each intersection point, there is an open neighborhood of the intersection point in which they intersect in the neighborhood after any small perturbation. For any set of hypercurves, we can homotope them so that they intersect transversely.

In [5], for a finite set $V$, Crisp and Wiest introduced a $V$-dissection on a compact (orientable or non-orientable) surface (see $[4,5,9,10]$ ). Let $V$ be a finite set. A $V$-dissection on $S$ is a set $\mathcal{H}$ of hypercurves satisfying: 
(1) Any two hypercurves of $\mathcal{H}$ either are disjoint or intersect transversely, and for any intersection point of two hypercurves, no other hypercurve than the two hypercurves passes through the intersection point.

(2) Each hypercurve in $\mathcal{H}$ is transversely oriented and labeled with an element of $V$ in such a way that any two hypercurves of the same label do not intersect.

We will call hypercurves of a dissection on $S$ dissection curves. A dissection curve labeled with $s$ is called a $s$-curve, while $s$ is called the label of the dissection curve. Likewise, for a subset $A$ of $V$, a dissection curve is called a $A$-curve, if the dissection curve is labeled with an element of $A$. Let $\gamma$ be a path in $S$. The content of $\gamma$ is the set of labels of dissection curves which intersect $\gamma$ and denoted by $\operatorname{cont}(\gamma)$. For a subsurface $S^{\prime}$ of $S$, the content of $S^{\prime}$ is the set of labels of dissection curves which intersect $S^{\prime}$ and denoted by $\operatorname{cont}\left(S^{\prime}\right)$.

Let $\Gamma$ be a finite simple graph. For convenience, a $V(\Gamma)$-dissection on $S$ will also be called as a $\Gamma$-dissection on $S$. Let $\mathcal{H}$ be a $\Gamma$-dissection on $S$. For a loop $\gamma:[0,1] \longrightarrow S$ based at a point $p \in S$, homotope $\gamma$ so that $\gamma$ intersect dissection curves transversely if they meet. We can define a word $w_{\gamma}$ to be the word in the letters from $\left\{v, v^{-1} \mid v \in V(\Gamma)\right\}$ obtained by reading off the labels of the hypercurves traversed by $\gamma$, where the sign of each letter is positive if $\gamma$ traverses with the transverse orientation of the dissection curve and the sign is negative, otherwise. We call the word $w_{\gamma}$ the label-reading of $\gamma$ with respect to the dissection. Since for any other $\gamma^{\prime}$ homotopic to $\gamma$, if $\gamma^{\prime}$ meets dissection curves transversely, two words $w_{\gamma}$ and $w_{\gamma}^{\prime}$ are equivalent in the free group generated by $V(\Gamma)$, we get a well-defined map $\psi: \pi_{1}(S, p) \longrightarrow A(\Gamma)$ defined by $\psi([\gamma])=w_{\gamma}$.

For two loops $l_{1}$ and $l_{2}$ with corresponding label-readings $w_{1}$ and $w_{2}$, the label-reading of the product of two paths $l_{1} * l_{2}$ is the concatenation $w_{1} w_{2}$ of the two words $w_{1}$ and $w_{2}$, thereby the map $\psi$ is a homomorphism. We call $\psi: \pi_{1}(S, p) \longrightarrow A(\Gamma)$ the homomorphism induced by a $\Gamma$-dissection on $S$. It is known that the converse also holds.

Proposition 2.1 ([5, 9, 10]). Let $S$ be a compact orientable surface (with or without boundary) and $\Gamma$ be a graph. For any homomorphism $\psi: \pi_{1}(S, p) \longrightarrow$ $A(\Gamma)$, there is a $\Gamma$-dissection on $S$ inducing $\psi$.

We say two maps $\phi, \psi: \pi_{1}(S) \longrightarrow A(\Gamma)$ are equivalent if $\phi=i \circ \psi$ for some inner-automorphism $i: A(\Gamma) \longrightarrow A(\Gamma)$. We say two dissections $\Delta$ and $\Delta^{\prime}$ on $S$ are equivalent if they induce equivalent homomorphisms $\pi_{1}(S, p) \longrightarrow A(\Gamma)$. Note that for a fixed dissection, a base change does not alter the equivalence class of the corresponding homomorphism. Certain simplifications of a given dissection on $S$ can be made without changing the equivalence class of the dissection as seen in the next proposition.

We say that two hypercurves $\alpha_{1}, \alpha_{2}$ in $S$ bound a bigon if there are subpaths $\alpha_{1}^{\prime}$ of $\alpha_{1}$ and $\alpha_{2}^{\prime}$ of $\alpha_{2}$ such that $\alpha_{1}^{\prime} \cup \alpha_{2}^{\prime}$ is the boundary of a disk on $S$. 
Proposition 2.2 ([5, 9, 10]). Let $\Delta$ be a $\Gamma$-dissection on a compact orientable surface $S$ with or without boundary. Let $\Delta^{\prime}$ be a $\Gamma$-dissection on $S$ obtained from $\Delta$ by the following simplifications:

(1) $\Delta^{\prime}$ is obtained by removing null-homotopic dissection curves in $\Delta$.

(2) $\Delta^{\prime}$ is obtained by removing properly embedded arcs in $\Delta$ that are homotopic into $\partial S$.

(3) Suppose that two dissection curves in $\Delta$ bound a bigon. By taking an innermost one, we may assume that the two curves do not touch the interior of the bigon. To get $\Delta^{\prime}$, we homotope the two curves in a neighborhood of the bigon so that the bigon is removed (Figure 1). Through the homotopy, the number of intersection points with other dissection curves in $\Delta$, labels and transverse orientations are not changed, while the number of intersection points of the two curves is reduced by two.

Then $\Delta$ and $\Delta^{\prime}$ are equivalent.
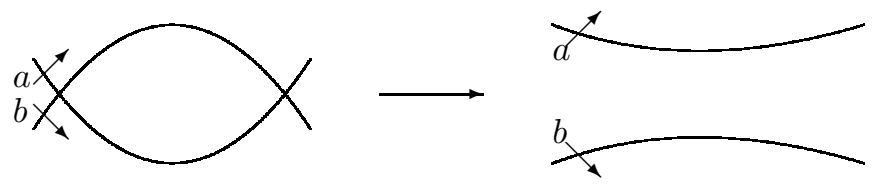

FiguRE 1. Removing a bigon

From now on, if there is no ambiguity, then we will call both simple closed curves and properly embedded arcs on a compact orientable surface as curves.

Two curves in a compact orientable surface are said to intersect minimally if either they does not intersect or they intersect transversely and do not bound a bigon. For curves $\alpha_{1}$ and $\alpha_{2}$ on a compact orientable surface, the intersection number $i\left(\alpha_{1}, \alpha_{2}\right)$ of $\alpha_{1}$ and $\alpha_{2}$ is defined as $\min \left\{\left|\alpha_{1}^{\prime} \cap \alpha_{2}^{\prime}\right| \in \mathbb{N} \mid\right.$ $\alpha_{i}^{\prime}$ is homotopic to $\alpha_{i}$ for each $\left.i=1,2\right\}$ (if $\alpha_{i}$ is a properly embedded arc, then the homotopy is relative to $\partial S$ ). It is known that two hypercurves $\alpha_{1}$ and $\alpha_{2}$ intersect minimally if and only if $\left|\alpha_{1} \cap \alpha_{2}\right|=i\left(\alpha_{1}, \alpha_{2}\right)$ ([2]).

Let $\alpha$ be a curve in a compact orientable surface $S$. Let $T$ be a compact subsurface of $S$. We say that $\alpha$ intersects $\partial T$ minimally if for any component $\partial_{i} T$ of the boundary $\partial T$ of $T, \alpha$ intersects $\partial_{i} T$ minimally.

For a finite collection of curves in a compact surface, we can homotope them so that any two curves in the set intersect minimally if they intersect (repeat the process in the Figure 1 successively). From now on, we assume that any dissection $\Delta$ on a compact surface $S$ satisfies the following (1), (2), and (3): 
(1) There is no null-homotopic dissection curve in $\Delta$.

(2) There is no properly embedded arc in $\Delta$ that is homotopic into $\partial S$.

(3) There is no bigon formed by dissection curves, that is, the dissection curves intersect minimally.

Now we prove a part of the basic cutting lemma in detail while Crisp, Sageev, and Sapir only sketched a proof of the lemma ([4]). For a set $B$ of curves in a compact surface $S$, each element of $B$ will be called a $B$-curve and $S-B$ will denote $S-\cup_{\gamma \in B} \gamma$. All curves are assumed to be homotoped to intersect minimally.

Suppose a graph $\Gamma$ is a union of connected induced subgraphs $\Gamma_{1}, \ldots, \Gamma_{n}$. Then $\Gamma$ is written as $\Gamma=\Gamma_{1} \cup_{L} \cdots \cup_{L} \Gamma_{n}$ if $L$ is an induced (not necessarily connected) subgraph of $\Gamma$ with $L=\Gamma_{i} \cap \Gamma_{j}$ for any distinct $i, j=1, \ldots, n$.

Lemma 2.3 (basic cutting lemma ([4])). Suppose that $\Gamma=\Gamma_{1} \cup_{L} \cdots \cup_{L} \Gamma_{n}(n \geq$ 2 ), and $S$ is a compact orientable surface equipped with a $\Gamma$-dissection $\Delta$. Then there exist collections $B_{1}, \ldots, B_{n}$ of essential simple closed curves and properly embedded arcs on $S$ as follows:

(1) All of the curves in $B_{1} \cup \cdots \cup B_{n}$ are mutually disjoint.

(2) Each $\gamma \in B_{i}$ is isotopic to a composition of subarcs of $\left(\Gamma_{i}-L\right)$-curves, where the isotopies are relative to $\partial S$ in the case that $\gamma$ is a properly embedded arc.

(3) Every closed curve on $S$ that intersects a curve from $B_{i}$ also intersects a $\left(\Gamma_{i}-L\right)$-curve from $\Delta$.

(4) If $S$ is a closed hyperbolic surface, then one of the components of $S-$ $\left(B_{1} \cup \cdots \cup B_{n}\right)$ has non-abelian fundamental group.

(5) For each $\gamma \in B_{i}$, $\operatorname{cont}(\gamma) \subset L$.

(6) If $S^{\prime}$ is a connected component of $S-\left(B_{1} \cup \cdots \cup B_{n}\right)$, then either $\operatorname{cont}\left(\overline{S^{\prime}}\right) \subset \Gamma_{i}$ for some $i=1, \ldots, n$ or $\operatorname{cont}\left(\overline{S^{\prime}}\right) \subset L$.

Proof. Set $\Gamma_{i}^{\prime}=\Gamma_{i}-L$ for each $i=1, \ldots, n$, and let $\Omega$ be the union of all $\Gamma_{i}^{\prime}$ curves on $S$. Note that $\Omega$ is not necessarily a simple graph. Take a closed regular neighborhood $N(\Omega)$ of $\Omega$. To $N(\Omega)$, attach every component of $\overline{S-N(\Omega)}$ which is a closed disk whose boundary does not share any arcs with $\partial S$ to obtain a compact surface $\widehat{S}$. Considering the structure of $\Gamma$, it is easy to observe that

(i) for each attaching disk, there is only one $i(i=1, \ldots, n)$ such that the attaching disk is surrounded by $\Gamma_{i}^{\prime}$-curves only in $S$;

(ii) for each component of $N(\Omega)$, there is only one $i$ such that the component contains $\Gamma_{i}^{\prime}$-curves only, and so it is the case for each component of $\widehat{S}$;

(iii) pasting closed disks of $\overline{S-N(\Omega)}$ to $N(\Omega)$ does not change the number of components, and so the number of components of $\widehat{S}$ is equal to the number of components of $N(\Omega)$. 
In view of (ii), $N(\Omega)$ has more than one components, as each $\Gamma_{i}^{\prime}$ is not empty. By (iii), $\widehat{S}$ is a disconnected compact surface with non-empty boundary. Since $S$ is connected, $\widehat{S} \varsubsetneqq S$.

For each $i=1, \ldots, n$, let $W_{i}$ be the union of all the components of $\widehat{S}$ containing a $\Gamma_{i}^{\prime}$-curve. Consider the collection $B_{i}$ of the simple closed curves and properly embedded arcs in $S$ obtained from $\partial W_{i}$ by removing the interior of $W_{i} \cap \partial S$. Note that $W_{i} \cap \partial S$ is the union of a finite number of subarcs of $\partial S$. Since $\Gamma_{i}^{\prime} \neq \emptyset, W_{i} \neq \emptyset$, and so $W_{i}$ is a compact orientable surface with non-empty boundary. It follows that $B_{i} \neq \emptyset$, as $\partial W_{i}$ is not contained in $\partial S$. It is easy to see that $B=B_{1} \cup \cdots \cup B_{n}$ is the collection of all the mutually disjoint simple closed curves and properly embedded arcs in $S$ obtained from $\partial \widehat{S}$ by removing the interior of $\widehat{S} \cap \partial S$.

Now (1), (2) and (3) are obvious by the construction of $B_{i}$-curves.

Suppose that $S$ is a closed surface. Then all dissection curves are simple closed curves. Note that $S-\left(B_{1} \cup \cdots \cup B_{n}\right)$ does not have any disk component, since every disk component $S-N(\Omega)$ is removed by attaching it to $N(\Omega)$ in the process of constructing $B$-curves. If all the components of $S-\left(B_{1} \cup \cdots \cup B_{n}\right)$ have abelian fundamental groups, they are annuli. Since an annulus attached to another annulus along one of its boundary circles becomes an annulus, $S$ must be a torus in this case, which is absurd. Therefore one of the components of $S-\left(B_{1} \cup \cdots \cup B_{n}\right)$ has non-abelian fundamental group. This proves (4).

Let $\gamma$ be a $B_{i}$-curve. Suppose a dissection curve $\alpha$ meets $\gamma$. The construction of $B$-curves guarantees that $\alpha$ can not be a $\Gamma_{k}^{\prime}$-curve for any $k=1, \ldots, n$. It follows that $\alpha$ must be $L$-curve. This proves (5).

Let $Y$ be the closure of a component of $S-\left(B_{1} \cup \cdots \cup B_{n}\right)$. Then $Y$ is either a component of $\widehat{S}$ or the closure of a component of $S-\widehat{S}$. If $Y_{1}$ is the closure of a component of $S-\widehat{S}$, then there is no $\Gamma_{i}^{\prime}$-curve in $Y_{1}$ for any $i=1, \ldots, n$, which means $Y_{1}$ meets $L$-curves only. Thus $\operatorname{cont}\left(Y_{1}\right) \subset L$. Now, let $Y_{2}$ be a component of $\widehat{S}$. Then $Y_{2}$ contains a $\Gamma_{i}^{\prime}$-curve for some $i$. It turns out that $\operatorname{cont}\left(Y_{2}\right) \subset \Gamma_{i}$. In fact, $Y_{2}$ touches no $\Gamma_{j}^{\prime}$-curve for $j \neq i$, as $\Gamma_{j}^{\prime}$-curve cannot intersect $\Gamma_{i}^{\prime}$-curve. Thus $Y_{2}$ touches $\Gamma_{i}$-curves only. This proves (6).

\section{Hyperbolic surface subgroups of right-angled Artin groups on proper subgraphs}

Crisp, Sageev, and Sapir obtained the following proposition in an effort to answer Question 1.2. Recall that for an induced subgraph $J$ of a graph $K$, the central HNN-extension $K *_{J}$ of $K$ over $J$ is obtained by adding a single vertex not belonging to $K$, and joining the new vertex to all the vertices of $J$ by edges.

Proposition 3.1 ([4]). Let $\Gamma$ be the amalgamation of two graphs $\Gamma_{1}$ and $\Gamma_{2}$ along a complete graph $L$. If $A(\Gamma)$ contains a hyperbolic surface subgroup, then $A\left(\Gamma_{1} *_{L}\right)$ or $A\left(\Gamma_{2} *_{L}\right)$ contains a hyperbolic surface subgroup. 
However, it is still not known whether either $A\left(\Gamma_{1}\right)$ or $A\left(\Gamma_{2}\right)$ contains a hyperbolic surface group. The easiest case to handle Question 1.2 is obviously the case where $L$ consists of only one vertex. It is known that hyperbolic surface groups do not contain the torus group. Thus two simple closed curves in a closed hyperbolic surface are homotopic if the elements represented by them in the fundamental group of the hyperbolic surface commutes. This enables us to homotope the curves not to intersect. This observation leads one to a positive answer to Question 1.2 in the case.

Lemma 3.2. Suppose that $\Gamma$ is a connected graph with $\Gamma=\Gamma_{1} \cup_{\Gamma_{1} \cap \Gamma_{2}} \Gamma_{2}$. Let $\Delta$ be a $\Gamma$-dissection on a closed surface $S$ other than the sphere inducing a monomorphism $\phi: \pi_{1}(S) \longrightarrow A(\Gamma)$. If $\Delta$ has no $\Gamma_{1} \cap \Gamma_{2}$-curves, then $\pi_{1}(S)$ embeds into either $A\left(\Gamma_{1}\right)$ or $A\left(\Gamma_{2}\right)$ by $\phi$.

Proof. Let $\Gamma_{i}^{\prime}=\Gamma_{1}-\left(\Gamma_{1} \cap \Gamma_{2}\right)$ for $i=1,2$. Since $\Delta$ has no $\Gamma_{1} \cap \Gamma_{2}$-curves, $\pi_{1}(S)$ embeds into the subgroup $A\left(\Gamma_{1}^{\prime}\right) * A\left(\Gamma_{2}^{\prime}\right)$ of $A(\Gamma)$ by $\phi$. Note that if the fundamental group of a closed surface is a subgroup of a free product of two non-trivial groups, then the group is a subgroup of one of the two factors. Therefore the conclusion follows.

Theorem 3.3. If $\Gamma$ is the graph amalgamation of two graphs $\Gamma_{1}$ and $\Gamma_{2}$ along a vertex and if $A(\Gamma)$ contains a hyperbolic surface group, then $A\left(\Gamma_{1}\right)$ or $A\left(\Gamma_{2}\right)$ contains a hyperbolic surface group.

Proof. Let $S$ be a closed hyperbolic surface and $\phi: \pi_{1}(S) \longrightarrow A(\Gamma)$ is a monomorphism. Let $\Delta$ be a $\Gamma$-dissection inducing $\phi$ and $\Gamma_{1} \cap \Gamma_{2}=\{a\}$. If $\Delta$ has only either $\Gamma_{1}$-curves or $\Gamma_{2}$-curves, then the conclusion follows. Thus we may assume that $\Delta$ has both $\left(\Gamma_{1}-\{a\}\right)$-curves and $\left(\Gamma_{2}-\{a\}\right)$-curves. By the previous lemma, we only need to show that $\Delta$ does not have $a$-curves.

Suppose $\Delta$ has a $a$-curve. Consider $B_{1}$-curves and $B_{2}$-curves as in the basic cutting lemma. Let $T$ be a component of $S-\left(B_{1} \cup B_{2}\right)$ (The component is not an open disk). Then $\bar{T} \subset S$ is a connected compact surface with nonempty boundary. Choose a boundary component $\partial_{1} \bar{T}$ of $\bar{T}$. By the basic cutting lemma, $\partial_{1} \bar{T}$ is a $B_{1}$-curve or a $B_{2}$-curve. By (5) in the basic cutting lemma, $\operatorname{cont}\left(\partial_{1} \bar{T}\right) \subset\{a\}$. Since $\phi$ is injective and $\partial_{1} \bar{T}$ is essential, $\operatorname{cont}\left(\partial_{1} \bar{T}\right)=$ $\{a\}$. Thus there is a dissection curve $\xi$ with label $a$ which intersects $\partial_{1} \bar{T}$ with positive intersection number. Then $\operatorname{cont}(\xi) \subset \operatorname{link}(a)$, and so $\phi([\xi])$ and $\phi\left(\left[\partial_{1} \bar{T}\right]\right)$ commutes in $A(\Gamma)$ where $[\xi],\left[\partial_{1} \bar{T}\right] \in \pi_{1}(S)$. Since $\phi$ is injective, $[\xi]$ and $\left[\partial_{1} \bar{T}\right]$ commutes in $\pi_{1}(S)$. Since $S$ is a closed orientable hyperbolic surface, $\xi$ and $\partial_{1} \bar{T}$ with proper choice of orientations are homotopic, and so their intersection number is 0 . Then $\xi$ can be homotoped so that $\xi \cap \partial_{1} \bar{T}=\emptyset$. This is a contradiction.

If we mimic the proof of Theorem 3.3, we can prove the following theorem.

Theorem 3.4. Let $\Gamma$ be the amalgamation of two graphs $\Gamma_{1}$ and $\Gamma_{2}$ along a graph L, Suppose that $\operatorname{link}(a)=\operatorname{link}(b)$ for any pair of vertices $a, b$ of $L$. If 
$A(\Gamma)$ contains a hyperbolic surface group, then either $A\left(\Gamma_{1}\right)$ or $A\left(\Gamma_{2}\right)$ contains a hyperbolic surface group.

Let $\Gamma$ be a graph which contains two vertices $a$ and $b$ with the same link. Suppose that $\Gamma-\{a, b\}$ is not connected. By applying Theorem 3.4 in the case when $L=\{a, b\}$, it can be shown that if $A(\Gamma)$ contains a hyperbolic surface group, then there is a component $K$ of $\Gamma-\{a, b\}$ such that the right-angled Artin group on the induced subgraph $K \cup\{a, b\}$ of $\Gamma$ contains a hyperbolic surface group.

In [1], Bell obtained a lemma which gives the underlying graph of the rightangled Artin group ker $\phi$, where $\phi$ is an epimorphism from a certain rightangled group to a finite cyclic group. This lemma provides us a useful tool to handle Question 1.1. Recall that the star of $v$, denoted by $\operatorname{star}(v)$, is the set $\{v\} \cup \operatorname{link}(v)$.

Lemma 3.5 (Bell's lemma [1]). Suppose $A(\Gamma)$ is a right-angled Artin group, and let $n$ be a positive integer. Choose a vertex $z \in V(\Gamma)$, and define $\phi$ : $A(\Gamma) \longrightarrow\left\langle x \mid x^{n}=1\right\rangle$ by $\phi(z)=x$ and $\phi(v)=1$ if $v \neq z$. Then $\operatorname{ker} \phi$ is the right-angled Artin group whose defining graph $\Gamma^{\prime}$ is obtained by gluing $n$ copies of $\Gamma-\{z\}$ to star $(z)$ along $\operatorname{link}(z)$. Moreover, the vertices of $\Gamma^{\prime}$ naturally correspond to the following generating set:

$$
\begin{aligned}
& \left\{z^{n}\right\} \cup \operatorname{link}(z) \cup\{u \mid u \notin \operatorname{star}(z)\} \cup\left\{z u z^{-1} \mid u \notin \operatorname{star}(z)\right\} \cup \cdots \\
& \cup\left\{z^{n-1} u z^{1-n} \mid u \notin \operatorname{star}(z)\right\} .
\end{aligned}
$$

Theorem 3.6. Let a be a vertex of a graph $\Gamma$. Suppose there are two vertices $b$ and $v$ other than $a$ with $\operatorname{link}(a)=\operatorname{link}(b) \subset \operatorname{link}(v)$. Then the right-angled Artin group $A(\Gamma)$ embeds into a subgroup of $A(\Gamma-\{a\})$. Therefore, if the rightangled Artin group $A(\Gamma)$ contains a hyperbolic surface subgroup, then $A(\Gamma-\{a\})$ contains a hyperbolic surface subgroup.

Proof. Let $X$ be the graph $\Gamma-\{a\}$. Then by applying Bell's lemma when $n=2$ to $X$, we see that if the graph $X^{\prime}$ is obtained by gluing two copies of $X-\{v\}$ to $\operatorname{star}(v)$ along $\operatorname{link}(v), A\left(X^{\prime}\right)$ embeds into $A(X)$. A copy of $X-\{v\}$ with $b$ in the other copy together forms an induced subgraph of $X^{\prime}$ which is isomorphic to $\Gamma$, as $b$ in the other copy plays the same role as $a$ in $\Gamma$. The conclusion follows.

Theorem 3.7. Let $\Gamma$ be a graph which contains two vertices $a$ and $b$ with the same link, where the number of vertices of the link $\leq 2$. If the rightangled Artin group $A(\Gamma)$ contains a hyperbolic surface subgroup, then $A(\Gamma-\{a\})$ contains a hyperbolic surface subgroup. Consequently, being a group isomorphic to $A(\Gamma-\{a\}), A(\Gamma-\{b\})$ also contains a hyperbolic surface group.

Proof. Let $L=\operatorname{link}(a)=\operatorname{link}(b)$. Then $\Gamma$ is an amalgamation of an induced (maybe disconnected) subgraph $\Gamma_{1}=\Gamma-\{a, b\}$ and the induced subgraph 
generated by $L \cup\{a, b\}$ along $L$. In the case where $L$ is one vertex, then the conclusion follows by Theorem 3.3.

Now consider the case where the number of vertices of $L$ is 2 . Let $L=$ $\{p, q\}$. First, assume that $L$ is complete. The induced subgraph $\langle a, b, p, q\rangle *_{L}$ of $\Gamma$ generated by $L \cup\{a, b\}$ is chordal, the right-angled Artin group defined on the graph does not contain any hyperbolic surface subgroup (Figure 2). By Proposition 3.1, the right-angled Artin group defined on the graph $\Gamma_{1} *_{L}$ contains a hyperbolic surface subgroup. Since $\Gamma_{1} *_{L}$ is isomorphic to $\Gamma-\{a\}$, the conclusion follows.

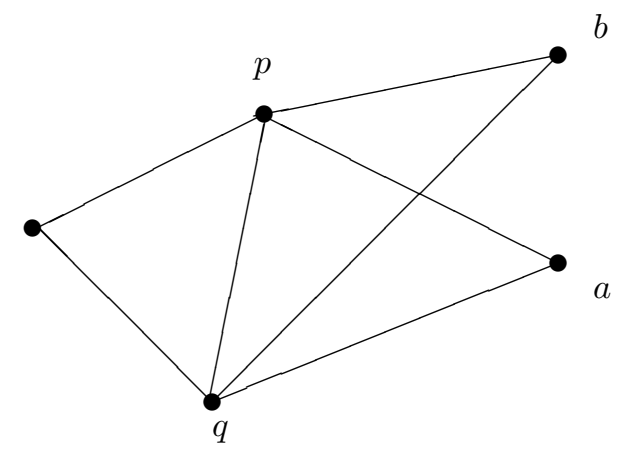

Figure 2. $\langle a, b\rangle *_{L}$

Suppose that $L$ is discrete. Then the distance $d(p, q)$ between $p$ and $q$ in $\Gamma_{1}$ is $\geq 2$. If $p$ and $q$ are disconnected in $\Gamma_{1}$, then by applying Theorem 3.3 twice, we see that the hyperbolic surface subgroup of $A(\Gamma)$ lies in $A\left(\Gamma_{1}\right)$. If $d(p, q)=2$, then there is a vertex $v$ in $\Gamma_{1}$ with $\operatorname{link}(a)=\operatorname{link}(b) \subset \operatorname{link}(v)$. By Theorem 3.6, $A(\Gamma-\{a\})$ contains a hyperbolic surface. If $d(p, q) \geq 3$, then $\Gamma-\{a\}$ has a long cycle which has $p, b, q$ as its successive vertices. Hence $A(\Gamma-\{a\})$ contains a hyperbolic surface group.

The complement graph $\bar{\Gamma}$ of a graph $\Gamma$ is the graph such that its vertex set is the vertex set of $\Gamma$ and for each pair of vertices $a, b$ in $\bar{\Gamma}$, there is an edge having $a, b$ as endpoints in $\bar{\Gamma}$ if there is no edge having $a, b$ as endpoints in $\Gamma$.

The join $L_{1} \star \cdots \star L_{n}$ of graphs $L_{1}, \ldots, L_{n}$ is defined as $\overline{\overline{L_{1}} \amalg \cdots \amalg \overline{L_{n}}}$ where $\amalg$ denotes the disjoint union.

Let $\Gamma=\Gamma_{1} \cup_{L} \cdots \cup_{L} \Gamma_{n}$, where $L$ is a join $L_{1} \star \cdots \star L_{n}$ of a join of subgraphs $L_{1}, \ldots, L_{n}$. Let $\Gamma_{i}^{\prime}=\Gamma_{i}-L$. Suppose for any $v \in V(\Gamma)-V\left(\Gamma_{i}^{\prime}\right)$ which is adjoint to a vertex in $V\left(\Gamma_{i}^{\prime}\right), v$ is a vertex of $L_{i}$. Then we say that $\Gamma$ is almost a join of $\Gamma_{1}, \ldots, \Gamma_{n}$ over $L_{1}, \ldots, L_{n}$.

Proposition $3.8([4])$. Suppose that $\Gamma$ is almost a join of subgraphs $\Gamma_{1}, \ldots, \Gamma_{n}$ over $L_{1}, \ldots, L_{n}(n \geq 2)$. Let $\Delta$ be a faithful $\Gamma$-dissection diagram on a compact hyperbolic surface $\bar{S}$ (with or without boundary) such that $\operatorname{cont}(\partial S)$ is in $L$. Then $\operatorname{cont}(S) \subset \Gamma_{i}$ for some $i$. 
This proposition implies that if $\Gamma$ is almost a join of subgraphs $\Gamma_{1}, \ldots, \Gamma_{n}$ over $L_{1}, \ldots, L_{n}(n \geq 2)$ and if $S$ is a closed hyperbolic surface and there is a monomorphism $\phi$ from $\pi_{1}(S)$ to $A(\Gamma)$, then actually the map $\phi$ is a monomorphism from $\pi_{1}(S)$ to $A\left(\Gamma_{i}\right)$ for some $i=1,2, \ldots$. A vertex subset $X$ of a graph $\Gamma$ is called separating if $\Gamma-X$ is disconnected.

Proposition 3.9 ([4]). Suppose that a connected graph $\Gamma$ contains no long cycle and suppose that $\{a, b\}$ is a separating pair of non-adjacent vertices in $\Gamma$ such that $\Gamma=\Gamma_{1} \cup_{\{a, b\}} \Gamma_{2}$ for some two proper induced subgraphs $\Gamma_{1}, \Gamma_{2}$. If $A(\Gamma)$ contains a hyperbolic surface subgroup, then $A\left(\Gamma_{1}\right)$ contains a hyperbolic surface subgroup.

Theorem 3.10. Let $\Gamma$ be a graph having two vertices $a, b$ with the same link, where the link has 3 vertices and is discrete. If the right-angled Artin group $A(\Gamma)$ contains a hyperbolic surface subgroup, then $A(\Gamma-\{a\})$ contains a hyperbolic surface subgroup.

Proof. Let $L=\left\{p_{1}, p_{2}, p_{3}\right\}$ be the link of both $a$ and $b$. Then $\Gamma$ is the amalgamation of an induced (maybe disconnected) subgraph $\Gamma_{1}=\Gamma-\{a, b\}$ and the induced subgraph generated by $L \cup\{a, b\}$ along $L$. For $i, j$ with $1 \leq i<j \leq 3$, we will call a vertex adjacent to both $p_{i}$ and $p_{j}$ a vertex of type $p_{i j}$. For example, $z_{12}, z_{23} z_{13}$ in Figure 4 are vertices of type $p_{12}, p_{23}, p_{13}$, respectively.

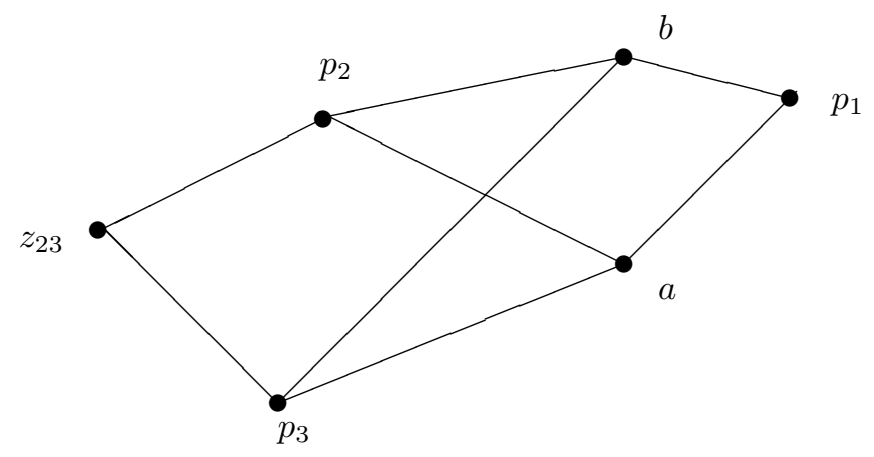

Figure 3. $p_{1}$ is not connected to $p_{2}, p_{3}$ by any edge path in $\Gamma_{1}$

First, consider the case where not all of the three points $p_{1}, p_{2}, p_{3}$ are in the same component in $\Gamma_{1}$. If any two of $p_{1}, p_{2}, p_{3}$ are not in the same component, then by applying Theorem 3.3 repeatedly, it can be easily shown that the right-angled Artin group defined on one of the components of $\Gamma_{1}$ contains a hyperbolic surface group, as Theorem 3.4 assures that the right-angled Artin group on the induced subgraph generated by $p_{1}, p_{2}, p_{3}, a, b$ can not contain a hyperbolic surface group. So, $A(\Gamma-\{a\})$ contains a hyperbolic surface group. 
Thus we may assume that only two of $p_{1}, p_{2}, p_{3}$ are in the same component in $\Gamma_{1}$. Suppose $p_{2}$ and $p_{3}$ are in the same component for convenience. If the distance between $p_{2}$ and $p_{3}$ is larger than 2 in $\Gamma_{1}$, then there is a long cycle. So, we only need to deal with the case of $d\left(p_{2}, p_{3}\right)=2$, where there is a vertex $z_{23}$ of type $p_{23}$. Then $\Gamma$ has the induced subgraph as in Figure 3 . Note that $\Gamma$ is almost a join of two subgraphs $K_{1}$ and $K_{2}$ over the graphs $\left\langle p_{2}, p_{3}\right\rangle$ and $\langle a, b\rangle$ where $K_{1}$ contains $\left\{z_{23}\right\}$ and $K_{2}$ contains $\left\{p_{1}\right\}$. By Proposition 3.8, either $A\left(K_{1}\right)$ or $A\left(K_{2}\right)$ contains a hyperbolic surface group. If $A\left(K_{1}\right)$ contains a hyperbolic surface subgroup, then by Theorem 3.7, $A\left(K_{1}-\{a\}\right)$ contains a hyperbolic surface subgroup. If $A\left(K_{2}\right)$ contains a hyperbolic surface subgroup, then by Theorem 3.3, $A\left(K_{2}-\left\{p_{2}, p_{3}, a, b\right\}\right)$ contains a hyperbolic surface subgroup as the right-angled Artin group on the induced subgraph generated by $p_{1}, p_{2}, p_{3}, a, b$ does not contain a hyperbolic surface group. Hence $A(\Gamma-\{a\})$ contains a hyperbolic surface group.

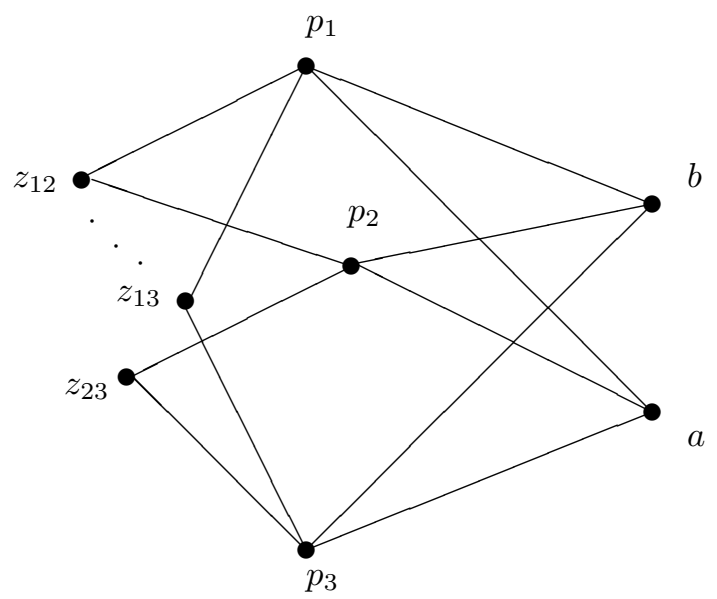

Figure $4 . \quad d\left(p_{1}, p_{2}\right)=d\left(p_{2}, p_{3}\right)=d\left(p_{1}, p_{3}\right)=2$ in $\Gamma_{1}$

Next, we need to deal with the case where $p_{1}, p_{2}, p_{3}$ are all in the same component in $\Gamma_{1}$. If there is a vertex $v$ which is of two different $p_{i j}$ types, then the vertex $v$ belongs to any of all three types. Thus $\operatorname{link}(a)=\operatorname{link}(b) \subset \operatorname{link}(v)$. By Theorem 3.6, $\Gamma-\{a\}$ contains a hyperbolic surface group. Therefore we may assume that there is no vertex of two different $p_{i j}$ types. First, we consider the case where $d\left(p_{1}, p_{2}\right)=d\left(p_{2}, p_{3}\right)=d\left(p_{3}, p_{1}\right)=2 \mathrm{in} \Gamma_{1}$. If any pair of vertices of different $p_{i j}$ types are not connected in $\Gamma_{1}-\left\{p_{1}, p_{2}, p_{3}\right\}$, there is an induced 6 -cycle in $\Gamma_{1}$. For example, in Figure $4, p_{1} z_{12} p_{2} z_{23} p_{3} z_{31} p_{1}$ is an induced 6-cycle, where $z_{12}, z_{23} z_{13}$ are vertices of types $p_{12}, p_{23}, p_{13}$, respectively. It follows that $\Gamma-\{a\}$ contains an induced 6-cycle. 
Now suppose there is a pair of vertices of different $p_{i j}$ types which is connected in $\Gamma_{1}-\left\{p_{1}, p_{2}, p_{3}\right\}$. Without loss of generality, we assume that a vertex $z_{12}$ of type $p_{12}$ is connected to a vertex of different $p_{i j}$ type by an edge path $\gamma$ (Figure 5). By deleting redundant vertices of $p_{12}$ type at the initial part of the edge path $\gamma$, we may assume that the second vertex among the vertices of $p_{i j}$ types on $\gamma$ is of different type, say $p_{13}$ type. Let $z_{13}$ be the second vertex. If each vertex on $\gamma$ except $z_{12}$ and $z_{13}$ is not adjacent to any of $p_{1}, p_{2}, p_{3}$, then $z_{12} \cdots z_{13} p_{3} b p_{2} z_{12}$ is an induced long cycle, as we can see in Figure 4 . Suppose among the vertices on $\gamma$, there is a vertex which is adjacent to only one of $p_{2}$ and $p_{3}$. Choose the nearest one $w$ to $z_{12}$ among such vertices. If $w$ is adjacent to $p_{3}$, then $z_{12} \cdots w p_{3} b p_{2} z_{12}$ is an induced long cycle. If $w$ is adjacent to $p_{2}$, then choose the nearest one $w^{\prime}$ among the vertices on $\gamma$ which is adjacent to $p_{3}$. By eliminating redundant vertices adjacent to $p_{2}$, we may assume that on $\gamma$, there is no vertex adjacent to only one of $p_{2}$ and $p_{3}$ between $w$ and $w^{\prime}$. In this case, $w \cdots w^{\prime} p_{3} b p_{2} w$ is an induced long cycle.

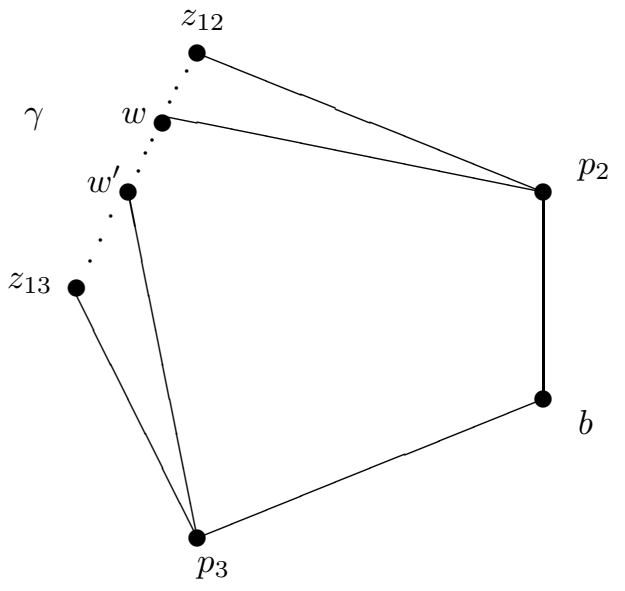

FigURE 5. $\quad \gamma$ with vertices adjacent to only one of $p_{2}$ and $p_{3}$

Now, suppose that $d\left(p_{i}, p_{j}\right) \geq 3$ in $\Gamma_{1}$ for some $p_{i}$ and $p_{j}$. Assume without loss of generality that $d\left(p_{1}, p_{3}\right) \geq 3$ in $\Gamma_{1}$. If there is an edge path from $p_{1}$ to $p_{3}$ realizing the distance $d\left(p_{1}, p_{3}\right)$ in $\Gamma_{1}$ and not passing through $p_{2}$, then the edge path from $p_{1}$ to $p_{3}$ and the edge path $p_{3} b p_{1}$ forms an induced long cycle together in $\Gamma-\{a\}$. Thus we may assume that for any edge path from $p_{1}$ to $p_{3}$ realizing the distance in $\Gamma_{1}$, the edge path passes through $p_{2}$. If either $d\left(p_{1}, p_{2}\right)$ or $d\left(p_{2}, p_{3}\right)$ is larger than 2 in $\Gamma_{1}$, then it is easy to see that there is an induced long cycle in $\Gamma-\{a\}$. For example, if $d\left(p_{1}, p_{2}\right)>2$, the edge path realizing the distance $d\left(p_{1}, p_{2}\right)$ and the edge path $p_{2} b p_{1}$ forms an induced long 


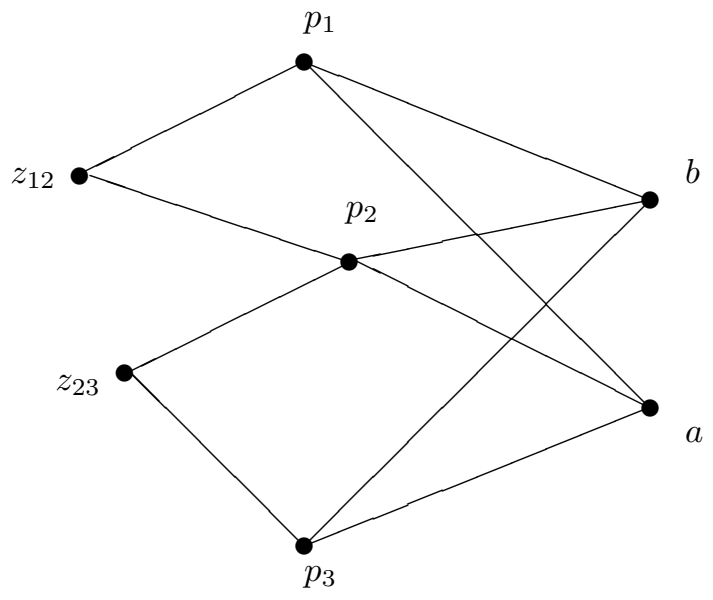

Figure $6 . \quad d\left(p_{1}, p_{2}\right)=2, d\left(p_{2}, p_{3}\right)=2, d\left(p_{1}, p_{3}\right)=4$ in $\Gamma_{1}$

cycle together in $\Gamma-\{a\}$. So, we only need to consider the case where $\Gamma$ has an induced subgraph as in Figure 6.

Note that in Figure 6, $\left\{p_{1}, p_{2}\right\}$ and $\left\{p_{2}, p_{3}\right\}$ are separating pairs. If $\Gamma-\{a\}$ contains a long cycle, it is done. Thus we may assume that $\Gamma-\{a\}$ does not have an induced long cycle. Then $\Gamma$ should not contain an induced long cycle. In fact, if $\Gamma$ contains an induced long cycle $C$, then $C$ contains $a$. Since $\operatorname{link}(a)=\operatorname{link}(b), C$ can not contain both $a$ and $b$, and so $C$ does not pass through $b$. If we replace $a$ with $b$ in $C$, we get another induced long cycle, which is in $\Gamma-\{a\}$. This is a contradiction. Thus $\Gamma$ does not contain an induced long cycle. Since $\left\{p_{1}, p_{2}\right\}$ and $\left\{p_{2}, p_{3}\right\}$ are separating pairs in $\Gamma$, by applying Proposition 3.9 twice, we see that $A(\Gamma-\{a, b\})$ contains a hyperbolic surface subgroup, as the right-angled Artin group on the induced subgraph generated by $p_{1}, p_{2}, p_{3}, a, b$ does not contain a hyperbolic surface group.

Corollary 3.11. Let $\Gamma$ be a graph having two vertices $a, b$ with the same link where the number of vertices of the link is $\leq 4$ and the link is discrete. Suppose that $\Gamma-\{a, b\}$ is disconnected. If the right-angled Artin group $A(\Gamma)$ contains a hyperbolic surface subgroup, then $A(\Gamma-\{a\})$ contains a hyperbolic surface subgroup.

Proof. Let $L=\operatorname{link}(a)=\operatorname{link}(b)=\left\{p_{1}, p_{2}, p_{3}, p_{4}\right\}$. Since $\Gamma-\{a, b\}$ is disconnected, $\Gamma$ can be considered as the amalgamation of two graphs $\Gamma_{1}$ and $\Gamma_{2}$ along $\{a, b\}$. Obviously, not all of the vertices of $L$ belong to the same $\Gamma_{i}(i=1,2)$ (Figure 7). Theorem 3.4 implies that either $A\left(\Gamma_{1}\right)$ or $A\left(\Gamma_{2}\right)$ contains a hyperbolic surface group. Since $\operatorname{link}(a)(=\operatorname{link}(b))$ in both $\Gamma_{1}$ and $\Gamma_{2}$ 


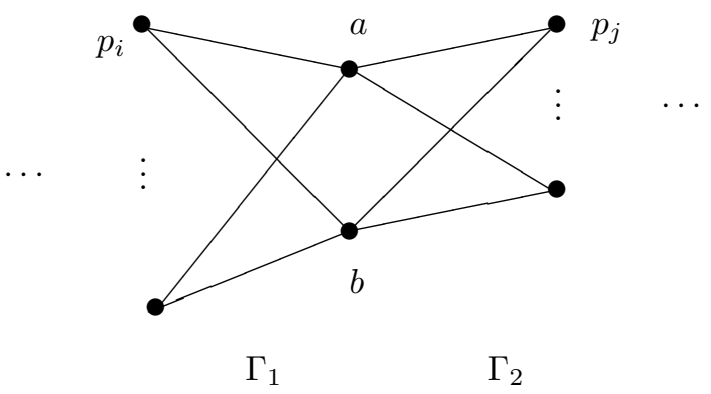

FIGURE 7. $p_{i}$ and $p_{j}$ are contained in different components of $\Gamma_{1}$

contains $\leq 3$ vertices, we can apply Theorem 3.10 and Theorem 3.7 to derive the conclusion.

The conclusion of Corollary 3.11 holds for a wider class of graphs. In fact, if we remove the restriction on the number of elements in $\operatorname{link}(a)=\operatorname{link}(b)$ and replace the condition that $\Gamma-\{a, b\}$ is disconnected with the condition that each component of $\Gamma-\{a, b\}$ contains less than 4 points of $\operatorname{link}(a)$, then the conclusion of Corollary 3.11 holds. In other words, let graph $\Gamma$ be a graph having two vertices $a, b$ with the same link. Suppose that the link is discrete and each component of $\Gamma-\{a, b\}$ contains less than 4 points of $\operatorname{link}(a)$. If $A(\Gamma)$ contains a hyperbolic surface group, then $A(\Gamma-\{a\})$ does so. A slight modification of the proof of Corollary 3.11 enables us to prove this.

\section{References}

[1] R. W. Bell, Combinatorial methods for detecting surface subgroups in right-angled Artin groups, preprint, 2010, available at: http://arXiv.org/abs/1012.4208.

[2] A. Casson and S. Bleiler, Automorphisms of Surfaces after Nielsen and Thurston, LMS Student Texts 9, Cambridge University Press, Cambridge, 1988.

[3] R. Charney, An introduction to right-angled Artin groups, Geom. Dedicata 125 (2007), 141-158.

[4] J. Crisp, M. Sageev, and M. Sapir, Surface subgroups of right-angled Artin groups, Internat. J. Algebra Comput. 18 (2008), no. 3, 441-491.

[5] J. Crisp and B. Wiest, Embeddings of graph braid and surface groups in right-angled Artin groups and braid groups, Algebr. Geom. Topol. 4 (2004), 439-472.

[6] C. Droms, Isomorphisms of graph groups, Proc. Amer. Math. Soc. 100 (1987), no. 3, $407-408$.

[7] J. Gross and T. W. Tucker, Topological Graph Theory, A Wiley-Interscience Publication, John Wiley and Sons, 1987.

[8] J. Kim, Hyperbolic Surface Subgroups of Right-Angled Artin Groups and Coxeter Groups, PhD thesis, Konkuk University, 2011. 
[9] S. Kim, Hyperbolic Surface Subgroups of Right-Angled Artin Groups and Graph Products of Groups, PhD thesis, Yale University, 2007.

[10] _ On right-angled Atrin groups without surface subgroups, Groups Geom. Dyn. 4 (2010), no. 2, 275-307.

[11] R. C. Lyndon and P. E. Schupp, Combinatorial Group Theory, Springer, Berlin, Heidelberg, New York, 1977.

[12] H. Servatius, C. Droms, and B. Servatius, Surface subgroups of graph group, Proc. Amer. Math. Soc. 106 (1989), no. 3, 573-578.

JongtaE KIM

Department of Mathematics

KonKuK University

Seoul 143-701, Korea

E-mail address: jtkim0731@gmail.com

Myoungho Moon

Department of Mathematics

KonKuK University

SeOul 143-701, Korea

E-mail address: mhmoon@konkuk.ac.kr 